Proceedings of the Edinburgh Mathematical Society (2003) 46, 373-378 (C)

DOI:10.1017/S0013091500001188 Printed in the United Kingdom

\title{
OPTIMAL EMBEDDINGS OF DISTRIBUTIONS INTO ALGEBRAS
}

\author{
H. VERNAEVE \\ Department of Pure Mathematics and Computer Algebra, University of Ghent, \\ Galglaan 2, B-9000 Gent, Belgium (hvernaev@cage.rug.ac.be)
}

(Received 27 November 2000)

\begin{abstract}
Let $\Omega$ be a convex, open subset of $\mathbb{R}^{n}$ and let $\mathcal{D}^{\prime}(\Omega)$ be the space of distributions on $\Omega$. It is shown that there exist linear embeddings of $\mathcal{D}^{\prime}(\Omega)$ into a differential algebra that commute with partial derivatives and that embed $\mathcal{C}^{\infty}(\Omega)$ as a subalgebra. This embedding appears to be the first one after Colombeau's to possess these properties. We show that many nonlinear operations on distributions can be defined that are not definable in the Colombeau setting.
\end{abstract}

Keywords: generalized functions; distributions; embeddings; injective modules; Colombeau

2000 Mathematics subject classification: Primary 46F30

Secondary $13 \mathrm{C} 11$

\section{Introduction}

Throughout this paper, $\Omega$ will be a convex, open subset of $\mathbb{R}^{n}$. Let $\mathcal{A}:=\left(\mathcal{C}^{\infty}(\Omega)\right)^{(0, \infty)}$ be the algebra of all families of $\mathcal{C}^{\infty}(\Omega)$-functions, indexed by elements of $(0, \infty)$. Let $I_{E}:=$ $\left\{\left(u_{\varepsilon}\right)_{\varepsilon>0} \in \mathcal{A}:\right.$ for all compact $K \subset \Omega$ there is $\varepsilon_{0}>0$ such that $u_{\varepsilon}(x)=0$ for all $\varepsilon<$ $\varepsilon_{0}$ and $\left.x \in K\right\}$. This ideal was introduced by Egorov in $[\mathbf{3}]$. It is derivative invariant (i.e. $\partial_{i}\left(I_{E}\right) \subseteq I_{E}$ for all partial derivatives $\partial_{i}$ ), and therefore $\mathcal{A} / I_{E}$ is a differential algebra.

We will embed $\mathcal{D}^{\prime}(\Omega)$ in the differential algebra $\mathcal{A} / I_{E}$. As described in [5, Chapter 6], it is well known how to construct embeddings in this algebra that ensure consistency with distributional derivatives or with products of $\mathcal{C}^{\infty}(\Omega)$-functions, but not both at the same time. Our embedding will be optimal in the sense that it has all these consistency properties.

Our construction starts from an embedding $\Phi$ of $T \in \mathcal{D}^{\prime}(\Omega)$ into $\mathcal{A} / I_{E}$ that commutes with partial derivatives. We modify $\Phi$ by inserting a map $F: \mathcal{D}^{\prime}(\Omega) \rightarrow \mathcal{C}^{\infty}(\Omega)$, i.e. we embed $T$ as the class of $F(T)+\Phi(T-F(T))$ in $\mathcal{A} / I_{E}$. It is not hard to see that, in order to embed $\mathcal{C}^{\infty}(\Omega)$ as a subalgebra without disturbing the consistency with distributional derivatives of $\Phi$, it suffices that $F$ is linear, commutes with partial derivatives and induces the identity on $\mathcal{C}^{\infty}(\Omega)$. In the next section, we will show the existence of such maps $F$ by algebraic arguments. We conclude by exhibiting some properties of this embedding that are lacking in Colombeau's embedding. 
We have tried to make the proofs in this article self-contained, avoiding the need for prior knowledge of either module theory or Rosinger's general theory of embedding distributions. The connections with these theories are added as remarks. Although the embedding was originally constructed in a non-standard setting, we have avoided the use of non-standard terminology.

\section{Smooth-part maps}

The first aim is to prove the existence of (non-injective) linear maps $F: \mathcal{D}^{\prime}(\Omega) \rightarrow \mathcal{C}^{\infty}(\Omega)$ that commute with all partial derivatives $\partial_{i}$ and that leave elements of $\mathcal{C}^{\infty}(\Omega)$ invariant. We will call such $F$ a smooth-part map.

In this section, we work in a more general context: let $V$ be a vector space over a field $\mathbb{K}$ and let $W$ be a subspace of $V$ (notation: $W \leqslant V$ ). Let $d_{1}, \ldots, d_{n}: V \rightarrow V$ be linear maps which commute mutually (i.e. $d_{i} \circ d_{j}=d_{j} \circ d_{i}, \forall i, j$ ). Then in this setting we call any linear map $F: V \rightarrow W$ that commutes with $d_{1}, \ldots, d_{n}$ and that leaves elements of $W$ invariant a smooth-part map.

Since $d_{1}, \ldots, d_{n}$ commute mutually, it makes sense to consider polynomials $p(d):=$ $p\left(d_{1}, \ldots, d_{n}\right) \in \mathcal{D}:=\mathbb{K}\left[d_{1}, \ldots, d_{n}\right]$ (i.e. in the variables $d_{i}$ and with coefficients in $\mathbb{K}$ ).

Definition. Consider systems of the form $p_{j}(d) u=v_{j}(j=1, \ldots, m)$, with $p_{j} \in \mathcal{D}$ and $v_{j} \in V$. Such a system is called compatible if and only if

$$
\forall q_{j}(d) \in \mathcal{D}: \sum q_{j}(d) p_{j}(d)=0 \Longrightarrow \sum q_{j}(d) v_{j}=0 .
$$

Compatibility is clearly a necessary condition for solvability of the system. $V$ is called an injective $\mathcal{D}$-module if any compatible system has a solution $u \in V$.

Lemma 2.1 ('induction' step). Suppose that $d_{i}(W) \leqslant W$ for each $i$, and that $W$ is an injective $\mathcal{D}$-module. Let $W \leqslant A \leqslant V$ and $d_{i}(A) \leqslant A$. Let $F: A \rightarrow W$ be a smoothpart map. Let $x \in V \backslash A$. Then there exists $B \leqslant V$ with $A \leqslant B, x \in B$ and $d_{i}(B) \leqslant B$ on which $F$ can be extended into a smooth-part map $G: B \rightarrow W$.

Proof. We call $I:=\{p(d) \in \mathcal{D}: p(d) x \in A\}$. Since $A$ is a linear subspace and $d_{i}(A) \leqslant A, I$ is easily seen to be an ideal of $\mathcal{D}$. Since polynomial rings in a finite number of variables over a field are Noetherian, there exists a finite number of generators for $I$, say $p_{1}, \ldots, p_{m}$. If $G$ exists, $G(x)$ must certainly satisfy $p_{j}(d) G(x)=F\left(p_{j}(d) x\right)(j=1, \ldots, m)$. Since $W$ is supposed to be an injective $\mathcal{D}$-module, this system has a solution in $W$ as soon as the compatibility condition is satisfied. This is the case, indeed

$$
\text { if } \sum q_{j}(d) p_{j}(d)=0, \quad \text { then } \sum q_{j}(d) F\left(p_{j}(d) x\right)=F\left(\sum q_{j}(d) p_{j}(d) x\right)=0 .
$$

We show that it is also sufficient to choose $G(x)$ as an arbitrary solution of this system. Let $B:=\{p(d) x+v: v \in A, p(d) \in \mathcal{D}\}$. Then $B$ clearly satisfies the properties of the statement of this lemma. With $G(x)$ already defined, there is no other choice for $G$ than

$$
G(p(d) x+v):=p(d) G(x)+F(v) .
$$


Let us check that this definition is independent of the representation of the element in $B$. So, suppose that $p(d) x+v=0$. Then $p(d) x=-v \in A$ and $p(d) \in I$. Therefore, there exist $q_{1}(d), \ldots, q_{m}(d) \in \mathcal{D}$ such that $p(d)=\sum_{j=1}^{m} q_{j}(d) p_{j}(d)$. Then

$$
\begin{aligned}
p(d) G(x) & =\sum_{j=1}^{m} q_{j}(d) p_{j}(d) G(x)=\sum_{j=1}^{m} q_{j}(d) F\left(p_{j}(d) x\right) \\
& =F\left(\sum_{j=1}^{m} q_{j}(d) p_{j}(d) x\right)=F(p(d) x)=F(-v) .
\end{aligned}
$$

So $p(d) G(x)+F(v)=0$ and $G$ is well defined. It is now easy to see that $G$ is a smooth-part map $B \rightarrow W$ extending $F$.

Theorem 2.2. Suppose that $d_{i}(W) \leqslant W$ for each $i$, and that $W$ is an injective $\mathcal{D}$-module. Then there exist smooth-part maps $V \rightarrow W$.

Proof. Let $\mathcal{F}$ be the set of all smooth-part maps $A \rightarrow W$, where $A \leqslant V$ with $d_{i}(A) \leqslant$ $A, \forall i$. We want to apply Zorn's Lemma on $\mathcal{F}$ provided with the following natural ordering: for $F, G \in \mathcal{F}$, we say that $F \leqslant G$ if and only if $G$ is an extension of $F$. The identity on $W$ belongs to $\mathcal{F}$, so $\mathcal{F}$ is not empty. Furthermore, if $\left(F_{\lambda}\right)_{\lambda \in \Lambda}$ is a chain of elements of $\mathcal{F}$, there exists an upper bound in $\mathcal{F}$, namely the function which has as a graph the union of the graphs of all $F_{\lambda}$. So, by Zorn's Lemma, there exists a maximal element $F_{\max } \in \mathcal{F}$. By the previous lemma, it must be defined on the whole of $V$.

Corollary 2.3. There exist smooth-part maps $\mathcal{D}^{\prime}(\Omega) \rightarrow \mathcal{C}^{\infty}(\Omega)$.

Proof. Let $V:=\mathcal{D}^{\prime}(\Omega), W:=\mathcal{C}^{\infty}(\Omega), d_{i}:=\partial_{i}$ and $\mathbb{K}:=\mathbb{C}$. Clearly, $\partial_{i}\left(\mathcal{C}^{\infty}(\Omega)\right) \leqslant$ $\mathcal{C}^{\infty}(\Omega), \forall i$. From the theory of overdetermined systems of partial differential equations with constant coefficients by Ehrenpreis [4], it follows that $\mathcal{C}^{\infty}(\Omega)$ is an injective $\mathcal{D}$ module (recall that $\Omega$ is still supposed to be open and convex). Therefore, the theorem can be applied.

Remark. The term injective $\mathcal{D}$-module, which is introduced in this article as a property about systems of equations, is a more general algebraic concept (cf. [1]). We can consider a vector space $W$ on which mutually commutative maps $d_{i}$ are defined as a module over the ring $\mathcal{D}$, where the scalar multiplication $p(d) \cdot w$ is nothing else than $p(d)(w) \in W$. A $\mathcal{D}$-module homomorphism is then a $\mathbb{K}$-linear map which commutes with each $d_{i}$. Since, in our case, the ring $\mathcal{D}$ is a commutative, Noetherian ring, one can see that the property of solvability of compatible systems (over $W$ ) can be formulated in an abstract way as follows:

for each ideal $I$ of $\mathcal{D}$ and each $\mathcal{D}$-module homomorphism $f: I \rightarrow W$, there exists $u \in W$ such that $f(p)=p \cdot u, \forall p \in I$. 
It is a classical result in the theory of injective modules that this condition is equivalent to the following property:

for each $\mathcal{D}$-module $M$ and each sub-module $N$ of $M$, each $\mathcal{D}$-module homomorphism $u: N \rightarrow W$ can be extended into a $\mathcal{D}$-module homomorphism $M \rightarrow W$.

The existence of smooth-part maps can be considered to be a special case of this result (both using Zorn's Lemma in their proof).

Remark. Let $W \leqslant V$ be such that $d_{i}(W) \leqslant W$ for each $i$. A smooth-part map $V \rightarrow W$ exists if and only if $W$ has an algebraic complement $U$ in $V$ for which $d_{i}(U) \leqslant U, \forall i$.

Indeed, suppose $F: V \rightarrow W$ is a smooth-part map. Then $W=\{v \in V: F(v)=v\}$, and $U:=\{v-F(v): v \in V\}$ does the job. On the other hand, suppose $U$ is as in the statement, then the unique decomposition $v=u+w(v \in V$ arbitrarily, $u \in U, w \in W)$ defines a map $F: V \rightarrow W: F(v):=w$, which is a smooth-part map.

\section{The new embedding}

Let $\mathcal{A} / I_{E}$ be the differential algebra introduced in $\S 1$. Let $F$ be a smooth-part map $\mathcal{D}^{\prime}(\Omega) \rightarrow \mathcal{C}^{\infty}(\Omega)$. Let $\left(\phi_{\varepsilon}\right)_{\varepsilon>0} \in \mathcal{D}^{(0, \infty)}$ be a strict delta net, i.e.

$$
\begin{gathered}
\operatorname{supp}\left(\phi_{\varepsilon}\right) \rightarrow 0, \quad \text { as } \varepsilon \rightarrow 0, \\
\int \phi_{\varepsilon}=1, \quad \forall \varepsilon>0, \\
\int\left|\phi_{\varepsilon}\right| \text { is bounded independently of } \varepsilon,
\end{gathered}
$$

and let $\left(\chi_{\varepsilon}\right)_{\varepsilon>0} \in(\mathcal{D}(\Omega))^{(0, \infty)}$ be a unit net, i.e. for each compact $K \subset \Omega$, there is $\varepsilon_{0}$ such that $\chi_{\varepsilon}=1$ on $K$ for $0<\varepsilon<\varepsilon_{0}$. Then we define an embedding $\iota: \mathcal{D}^{\prime}(\Omega) \rightarrow \mathcal{A} / I_{E}$ as follows:

$$
\iota(T):=\left(F(T)+\left[(T-F(T)) \chi_{\varepsilon}\right] \star \phi_{\varepsilon}\right)_{\varepsilon>0}+I_{E},
$$

where $\star$ denotes the convolution on $\mathbb{R}^{n}$.

Theorem 3.1. $\iota$ is a linear embedding that commutes with partial derivatives and that embeds $\mathcal{C}^{\infty}(\Omega)$ as a subalgebra.

Proof. As $F$ is linear, so is $\iota$. Next, we prove injectivity. Let $\iota(T)=0$, so $\left(U_{\varepsilon}\right):=$ $\left(F(T)+\left[(T-F(T)) \chi_{\varepsilon}\right] \star \phi_{\varepsilon}\right)_{\varepsilon>0} \in I_{E}$ and, in particular, it converges to 0 in $\mathcal{D}^{\prime}(\Omega)$. But $\forall V \in \mathcal{D}^{\prime}(\Omega):\left(V \chi_{\varepsilon}\right) \star \phi_{\varepsilon} \rightarrow V$ in $\mathcal{D}^{\prime}(\Omega)$, so $U_{\varepsilon} \rightarrow T$ in $\mathcal{D}^{\prime}(\Omega)$ and $T=0$.

For $T \in \mathcal{C}^{\infty}(\Omega), F(T)=T$ and so $\iota(T)$ is the constant family $(T)_{\varepsilon>0}$. Therefore, $\mathcal{C}^{\infty}(\Omega)$ is embedded as a subalgebra. Finally, we have for each $i$

$$
\begin{aligned}
\partial_{i} \iota(T) & =\left(\partial_{i} F(T)+\partial_{i}\left[(T-F(T)) \chi_{\varepsilon}\right] \star \phi_{\varepsilon}\right)_{\varepsilon>0}+I_{E} \\
& =\left(F\left(\partial_{i} T\right)+\left[\left(\partial_{i}(T-F(T))\right) \chi_{\varepsilon}\right] \star \phi_{\varepsilon}\right)_{\varepsilon>0}+I_{E}=\iota\left(\partial_{i} T\right),
\end{aligned}
$$

since $F$ commutes with partial derivatives and $\left(\left[(T-F(T)) \partial_{i} \chi_{\varepsilon}\right] \star \phi_{\varepsilon}\right)_{\varepsilon>0}$ is in $I_{E}$. 
Remark. The embedding can be interpreted in the general framework of Rosinger. We then obtain the following commutative diagram (cf. $[\mathbf{5}, \mathbf{6}])$ :

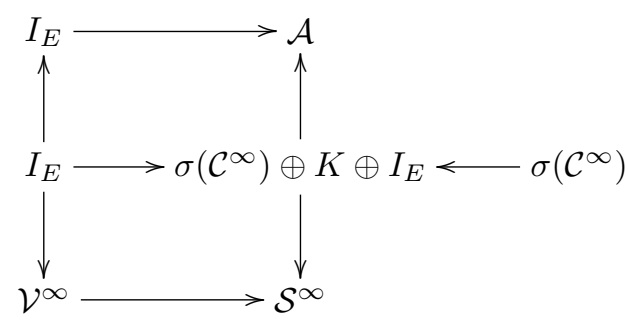

where

(i) $\sigma\left(\mathcal{C}^{\infty}\right)$ is the space of constant sequences $(u)_{\varepsilon>0} \in \mathcal{A}$,

(ii) $\mathcal{S}^{\infty}=\left\{\left(u_{\varepsilon}\right)_{\varepsilon>0} \in \mathcal{A}: u_{\varepsilon}\right.$ converges in $\left.\mathcal{D}^{\prime}(\Omega)\right\}$,

(iii) $\mathcal{V}^{\infty}=\left\{\left(u_{\varepsilon}\right)_{\varepsilon>0} \in \mathcal{A}: u_{\varepsilon} \rightarrow 0\right.$ in $\left.\mathcal{D}^{\prime}(\Omega)\right\}$, and

(iv) $K=\left\{\left(\left(w \chi_{\varepsilon}\right) \star \phi_{\varepsilon}\right)_{\varepsilon>0} \in\left(\mathcal{C}^{\infty}(\Omega)\right)^{(0, \infty)}: w \in U\right\}$, with $U$ an algebraic complement of $\mathcal{C}^{\infty}(\Omega)$ in $\mathcal{D}^{\prime}(\Omega)$ for which $\partial_{i}(U) \leqslant U, \forall i$.

\section{Comparison with Colombeau's embedding}

This new embedding appears to be the first one after Colombeau's which unifies both the commutation with partial derivatives and the embedding of $\mathcal{C}^{\infty}(\Omega)$ as a subalgebra. Both embeddings allow nonlinear operations on distributions to be defined. But in contrast with the Colombeau algebras, operators can be defined on $\mathcal{A} / I_{E}$ that do not preserve the moderate character or the nullness of the elements in the sense of Colombeau, e.g. for any $\mathcal{C}^{\infty}$-map $f: \mathbb{C}^{m} \rightarrow \mathbb{C}$ and any $u_{j}:=\left(u_{j, \varepsilon}\right)_{\varepsilon>0}+I_{E} \in \mathcal{A} / I_{E}(j=1, \ldots, m)$,

$$
f\left(u_{1}, \ldots, u_{m}\right):=\left(f\left(u_{1, \varepsilon}, \ldots, u_{m, \varepsilon}\right)\right)_{\varepsilon>0}+I_{E}
$$

is a well-defined element of $\mathcal{A} / I_{E}$, so any such $f$ gives rise to a well-defined map $\left(\mathcal{A} / I_{E}\right)^{m} \rightarrow \mathcal{A} / I_{E}$. This means that, by means of our embedding, the composition of $f$ with an $m$-tuple of distributions is defined in $\mathcal{A} / I_{E}$. In the Colombeau setting, growth conditions on $f$ are necessary (cf. [2]).

Acknowledgements. This work was supported by a grant from the University of Ghent, Bijzonder Onderzoeksfonds. The author thanks Professor C. Impens and Professor M. Oberguggenberger for useful discussions.

\section{References}

1. N. Bourbaki, Algèbre homologique, in Algèbre, ch. 10 (Masson, Paris, 1980).

2. J. F. Colombeau, New generalized functions and multiplication of distributions, Mathematical Studies, vol. 84 (North-Holland, Amsterdam, 1984). 
3. Y. V. Egorov, A contribution to the theory of generalized functions, Russ. Math. Surv. 45 (1990), 1-49 (transl. from Russian).

4. L. Ehrenpreis, Fourier analysis in several complex variables, Pure and Applied Mathematical Texts, vol. 17 (Wiley Interscience, New York, 1970).

5. M. OBergugGenberger, Multiplication of distributions and applications to partial differential equations, Pitman Research Notes in Mathematics, vol. 259 (Longman, Essex, 1992).

6. E. E. Rosinger, Nonlinear partial differential equations: sequential and weak solutions, Mathematical Studies, vol. 44 (North-Holland, Amsterdam, 1980). 